\title{
Article \\ Performance Entitlement by Using Novel High Strength Electrical Steels and Copper Alloys for High-Speed Laminated Rotor Induction Machines
}

\author{
Fengyu Zhang ${ }^{1}$, David Gerada ${ }^{1, *}$, Zeyuan Xu ${ }^{1}$, Yuling He ${ }^{2} \mathbb{D}$, He Zhang ${ }^{3}$, Wei Hua ${ }^{4}$ and Chris Gerada ${ }^{1}$ \\ 1 Power Electronics, Machines and Control Group, University of Nottingham, Nottingham NG7 2RD, UK \\ Fengyu.Zhang1@nottingham.ac.uk (F.Z.); Zeyuan.Xu@nottingham.ac.uk (Z.X.); \\ Chris.Gerada@nottingham.ac.uk (C.G.) \\ 2 Department of Mechanical Engineering, North China Electric Power University, Baoding 071003, China; \\ heyuling1@ncepu.edu.cn \\ 3 Key Laboratory of More Electric Aircraft Technology of Zhejiang Province, University of Nottingham Ningbo \\ China, Ningbo 315100, China; he.zhang@nottingham.edu.cn \\ 4 School of Electrical Engineering, Southeast University, Nanjing 210096, China; huawei1978@seu.edu.cn \\ * Correspondence: David.Gerada@nottingham.ac.uk
}

check for updates

Citation: Zhang, F.; Gerada, D.; Xu, Z.; He, Y.; Zhang, H.; Hua, W.; Gerada, C. Performance Entitlement by Using Novel High Strength Electrical Steels and Copper Alloys for High-Speed Laminated Rotor Induction Machines. Electronics 2022, 11, 210. https://doi.org/10.3390/ electronics11020210

Academic Editor: Domenico Casadei

Received: 30 August 2021

Accepted: 8 November 2021

Published: 10 January 2022

Publisher's Note: MDPI stays neutral with regard to jurisdictional claims in published maps and institutional affiliations.

Copyright: (C) 2022 by the authors. Licensee MDPI, Basel, Switzerland. This article is an open access article distributed under the terms and conditions of the Creative Commons Attribution (CC BY) license (https:// creativecommons.org/licenses/by/ $4.0 /)$.

\begin{abstract}
The laminated rotor Induction Machine (IM), with its simple construction and manufacturing, robustness, ease of control and comparatively lower cost remains by far the most utilized electromechanical energy converter. At very high speeds, traditionally its use is considered to be limited to the previously established operational limits of $2.5 \times 10^{5} \mathrm{rpm} \sqrt{ } \mathrm{kW}$, beyond which the surface Permanent Magnet (PM) Machine and the solid rotor Induction Machine become the machines available for consideration. The aforesaid limits are derived from the use of classic materials. This paper reviews the recent developments in electrical steels and copper alloys and translates these into the resulting performance entitlement and operational limits through a case study involving a marine application, for which an existing rare-earth PM machine is in use. It is concluded that with novel materials, laminated rotor induction machines can be operated up to $6 \times 10^{5} \mathrm{rpm} \sqrt{ } \mathrm{kW}$, thus opening the use of the rare-earth free Induction Machine for a wider application range previously limited to PM machines.
\end{abstract}

Keywords: high strength electrical steel; copper alloys; induction machines; high speed; operational limits

\section{Introduction}

The utilization of rotating mechanical machinery at high speeds has been in commercial use for at least the last 80 years and can be considered as mature technology. Application fields include superchargers, turbochargers and car racing engines amongst others, with rotational speeds over $10 \mathrm{krpm}$ and having an $\mathrm{rpm} \sqrt{ } \mathrm{kW}$ over $1 \times 10^{5}$ [1].

Aided by the rapid developments in power electronic (PE) devices that can switch at ever-higher frequencies, and corresponding PE converter topologies, the last two decades have seen unprecedented research, product development and market uptake of rotating high-speed electrical machines. Making electrical machines rotate at higher speeds melds well with technology roadmaps of increasing their volumetric/gravimetric power density as well as their cost-performance [2].

Ultimately, in many application areas, the market uptake of high-speed electrical machines primarily depends on the strength of the business case for the use of such machines with respect to the conventional use of systems that employ a lower-speed, geared machine. The business case is, in turn, strongly influenced by the electrical machine type. Due to the mechanical considerations and complexities, the electrical machine options for high speed are more limited. In [3], the various types of electrical machines suitable 
for high speed were investigated through an extensive review including machines that have been built and tested in industry and academia. The induction machine, in the solid and laminated versions, the switched reluctance machine and the surface permanent magnet machines are by far the most suitable types for high speed and occupy almost the entire high speed drive market. In the aforementioned study, it was established that different operational limits exist and a reliable figure of merit for different technologies is the $\mathrm{rpm} \sqrt{ } \mathrm{kW}$. Figure 1 summarizes the previously established limits.

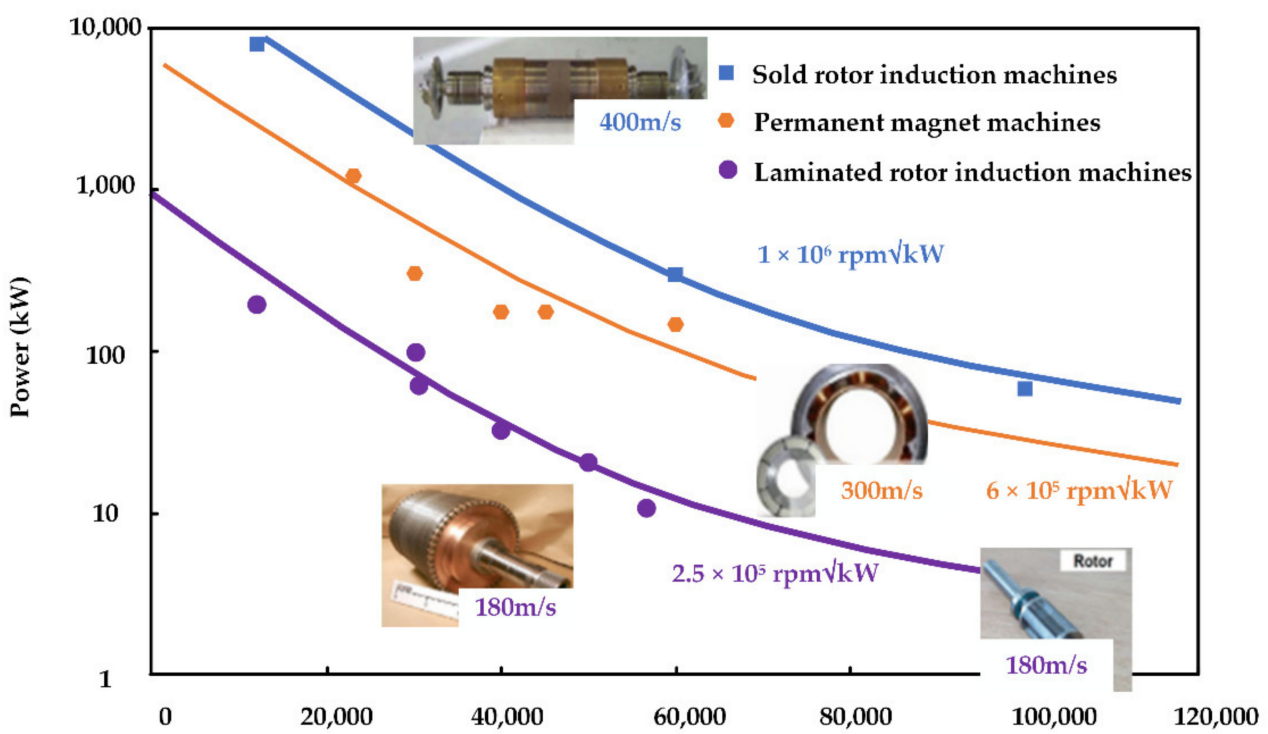

Figure 1. High speed electrical machine limits.

In this figure, the blue line represents the $\mathrm{rpm} \sqrt{ } \mathrm{kW}$ limit line of the solid rotor induction motor, which is approximately $1 \times 10^{6} \mathrm{rpm} \sqrt{ } \mathrm{kW}$. While the solid rotor induction machine achieves the highest power-speed capability, it suffers from comparatively lower efficiencies, due to the high eddy current losses generated within the solid body. While the performance can be improved by using a copper coating on top of the solid body, this also increases the magnetic airgap and hence reduces the power factor. In general, there is a consensus that for many applications, when possible, the use of a squirrel-cage laminated rotor induction machine is preferable due to the higher efficiencies and better power factor $[4,5]$; however, the achievable $\mathrm{rpm} \sqrt{ } \mathrm{kW}$ drops to around $2.5 \times 10^{5} \mathrm{rpm} \sqrt{ } \mathrm{kW}$, highlighted by the purple line in Figure 1, thus precluding its use for a wide range of applications that require an elevated power and speed rating, such as in the oil-gas industry, compressor markets, as well as within waste heat recovery systems for high horse power internal combustion engines, or other high speed applications that require a wide constant power speed range (CSPR) to be achieved, where the Induction Machine would have considerable system level advantages.

Finally, the third common topology for high-speed applications is the Surface mount Permanent Magnet Machine (SM-PMSM), in which magnets are typically mounted on a solid shaft and retained under compression through the use of a high-strength sleeve typically made of materials such as Inconel, titanium or carbon fiber. In this case, the traditional limit line, shown in orange is about $6 \times 10^{5} \mathrm{rpm} \sqrt{ } \mathrm{kW}$. Table 1 summarizes the aforesaid limits, as well as the limits of other topologies that have been used for high-speed applications, albeit less frequently. 
Table 1. Summary of high speed machine limits.

\begin{tabular}{cc}
\hline Machine Technology & $\mathbf{R p m} \sqrt{ } \mathbf{k W}$ \\
\hline Solid rotor IM & $1 \times 10^{6}$ \\
Laminated rotor IM with normal strength laminations & $2.5 \times 10^{5}$ \\
Switched Reluctance Motor & $3.5 \times 10^{5}$ \\
Surface PM with sleeve (no rotor laminations) & $6 \times 10^{5}$ \\
\hline
\end{tabular}

It should be noted that the limits presented in Figure 1 and Table 1 refer to the use of conventional materials. Recently, there have been relevant and interesting material developments both in the field of electrical steels, as well as in the field of copper alloys, with such materials potentially serving as an enabler to push the boundaries for high-speed laminated rotor induction machines. This paper is structured as follows: Section 2 reviews the developments in high strength copper alloys and electrical steels, Section 3 describes the multi-domain design considerations of a $150 \mathrm{~kW}$ high speed machine for an electrically assisted marine engine turbocharger, Section 4 discusses the development aspects and finally, the conclusions from this research are presented in Section 5.

\section{Development in Materials}

The constructional layout of an induction machine rotor is shown in Figure 2. Essentially, it consists of a laminated electrical steel stack with a plurality of slots, which are filled with bars made of a high electrical conductivity material (usually aluminum, copper, or a derived alloy) typically by a casting process, or by fabrication in case of low volumes. The bars are connected together (or 'short-circuited'), by means of end-rings, as shown in the same figure.

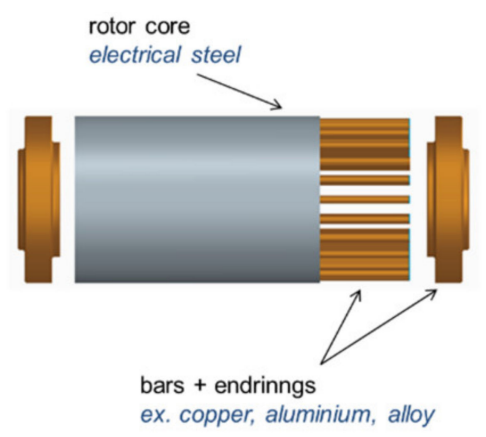

Figure 2. Constructional layout of a laminated rotor induction machine.

\subsection{Electrical Steels}

Table 2 summarizes the desired material properties when designing induction machines in order to achieve higher peripheral speeds, higher powers and consequently, higher values of $\mathrm{rpm} \sqrt{ } \mathrm{kW}$. High speed electrical machines employ high fundamental frequencies, and hence, for the stator, it is important to utilize electrical steels that have low iron losses, typically of a thin grade $(0.2 \mathrm{~mm}$ or less). For the rotor laminations, apart from the magnetic properties, it is desired to achieve higher yield strength, and good ductility, as any cracks in brittle materials will quickly propagate and translate to early failures. For a long time in the electromechanical industry, electrical steels were traditionally sold with guaranteed magnetic properties, i.e., magnetic permeability and core-losses corresponding to IEC60404. On the other hand, the mechanical properties of electrical steel were given secondary considerations, often with manufacturers stating 'typical' rather than 'guaranteed' values, giving a broad range of typical mechanical properties. From the authors' experience, often the aforesaid typical values vary significantly between one batch and another, at times with lower mechanical properties than those quoted as 'typical'. This, of course, creates a problem when designing and volume-producing high-speed electrical 
machines, and would necessitate the use of higher values of mechanical design margins, to account for the uncertainty in the material mechanical properties.

Table 2. Induction machine constituent materials and desired properties for high-speed IM.

\begin{tabular}{cc}
\hline Materials & Desired Properties \\
\hline Stator Laminations & Low loss, high saturation flux density \\
Rotor Laminations & High strength, good ductility \\
Rotor Cage Alloy & High strength, withstands high temperature, high electrical conductivity \\
\hline
\end{tabular}

Figure 3 maps the core losses at high frequency and yield strengths for various grades of SiFe and CoFe. The thickness of the grade and the \% elongation (measure of ductility) are also included.

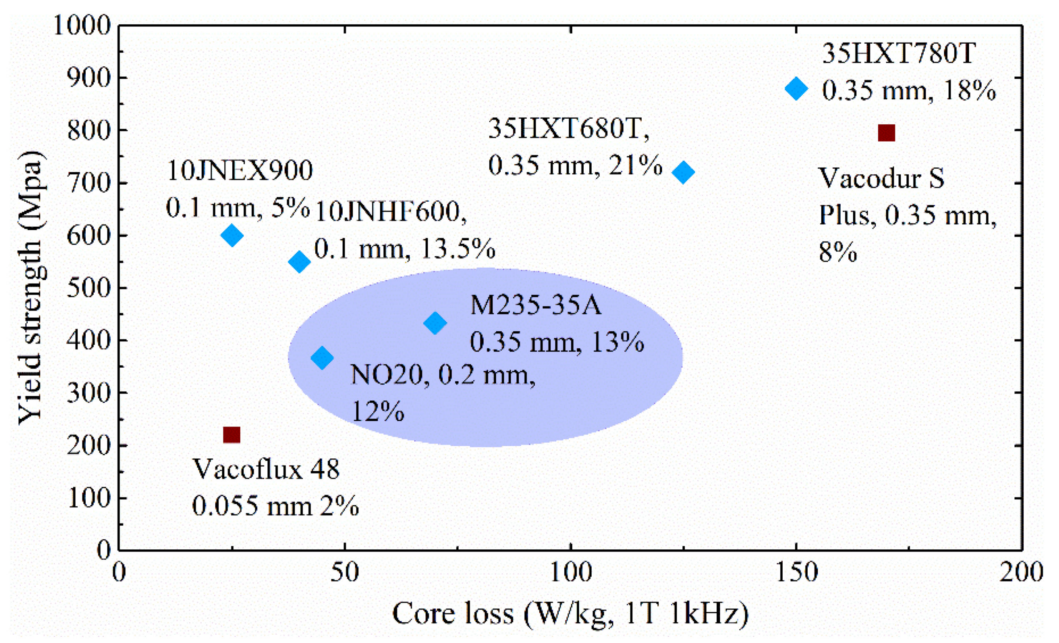

Figure 3. Conventional high-spec and new electrical steels.

For example, the grade M235-35A is a 0.35-millimeter-thick SiFe grade that is widely employed in earlier traction machines used with HEVs. Such a grade achieves a typical yield strength of about $400 \mathrm{MPa}$, with core losses at $1 \mathrm{~T} 1 \mathrm{kHz}$ of around $60 \mathrm{~W} / \mathrm{kg}$.

With the trend to move to higher frequencies, while achieving high efficiencies due to battery considerations, nowadays, there is a shift to employ 0.2-millimeter-thick grades in many mass-produced applications, such as NO20, which at the reference induction ( $1 \mathrm{~T}$, $1 \mathrm{kHz}$ ), reduce the core losses by around $25 \%$, at the cost of reduced mechanical strength. For the best-in-class SiFe grade (in terms of core losses), JNEX900, the silicon content is doubled [6], from 3 to $6 \%$, which reduces the losses down to $25 \mathrm{~W} / \mathrm{kg}$. The yield strength also increases in the process, although the higher amount of silicon makes the material more brittle.

For achieving outstanding mechanical properties for electrical steels used within high speed rotors, methods such as dislocation strengthening are used [7]. For example, the new grade 35HXT780T achieves a yield strength of $850 \mathrm{MPa}$ with improved ductility with respect to the other $\mathrm{SiFe}$ grades [8]. In the process of achieving the superior mechanical properties, the core losses are also increased. However, if the material is used solely for the rotor, its core losses properties are less of a concern. Indeed, the use of different grades of materials for the stator and rotor is recently increasingly common, thanks to the improvements in manufacturing, which reduce the material wastage.

\subsection{Copper Alloys}

The choice of material for the rotor cage of high-speed induction machines necessitates careful considerations. Pure copper is often not used due to its lower yield strength and the tendency to soften at higher temperatures. Different types of copper alloys can be considered, as shown in Figure 4, however, in general the stronger the alloy is, the less its 
electrical conductivity is, which increases the losses. With an induction machine, losses in the rotor are more difficult to dissipate. With many commercial high speed induction machines, operating to the limit line of Figure 1, often alloys of $\mathrm{CuAl}_{2} \mathrm{O}_{3}, \mathrm{CuCr}$ and $\mathrm{CuZr}$, with yield strengths of around 400-450 MPa, are used [9].

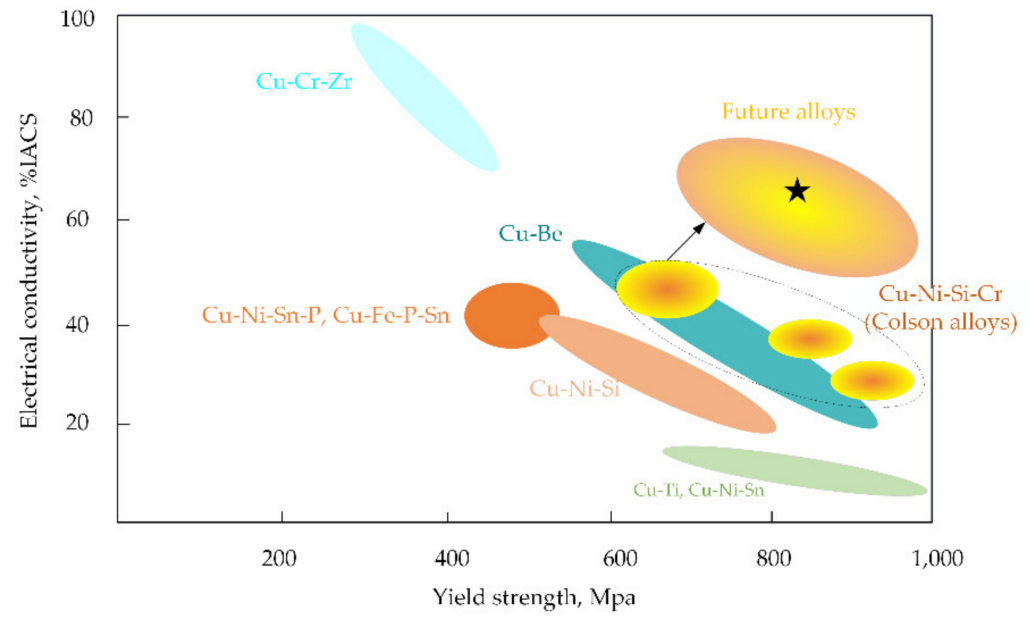

Figure 4. Conventional High-Spec and new copper alloys.

For higher strength, above $700 \mathrm{MPa}$, the material of choice has traditionally been $\mathrm{CuBe}$; however, it is well known that using this alloy is difficult due to Beryllium being a carcinogen, and its use and machining is only allowed in special circumstances with high levels of protection for those in close proximity. A recent interesting development are the so-called Colson alloys ( $\mathrm{CuNiSiCr}$ ) that achieve a similar performance to $\mathrm{CuBe}$, without the use of Beryllium, thus facilitating their implementation in a mass-production environment. Current research is looking at pushing the aforesaid alloys to ever higher performance, with strengths in excess of $900 \mathrm{MPa}$ and conductivities above 70\% IACS (International Annealed Copper Standard) [10].

\section{The Design of a $150 \mathrm{~kW} 50 \mathrm{krpm}$ Induction Machine Using Novel Materials}

Table 3 summarizes the requirement for the marine engine heat recovery application. The machine is $150 \mathrm{~kW}$, which needs to be achieved over a speed range of 25-50 krpm, thus translating to an $\mathrm{rpm} \sqrt{ } \mathrm{kW}$ of $6 \times 10^{5}$. The power-speed requirement derives from the needs of a $3 \mathrm{MW}$-class marine engine. Engine coolant ethylene-water-glycol (EWG) is available to cool the machine.

Table 3. Requirements for marine EAT (Electrically Assisted Turbocharger) machine for a diesel engine.

$\begin{array}{cc}\text { Power Rating } & 150 \mathrm{~kW} \\ \text { Coolant type/temperature } & \mathrm{EWG}, 100^{\circ} \mathrm{C}, 10 \mathrm{~L} / \mathrm{min} \\ \text { Speed } & 25-50 \mathrm{krpm} \\ \mathrm{rpm} \sqrt{ } \mathrm{kW} & 6 \times 10^{5} \\ \text { Maximum design frequency } & <1 \mathrm{kHz}\end{array}$

At the time of initiating this research, an existing surface PM solution was available, which, although fully functional, makes a difficult case due to the mass (and cost) of the magnets involved, and hence it was desired to move to a magnet-free, induction machine solution. Given that the aim of the application is higher fuel efficiencies, preference was to be made to higher efficiency, laminated rotor solutions if possible. While it is known that with conventional materials such a power-speed combination is outside the operational territory of laminated rotor induction machines, this research investigates as to whether 
the aforesaid $\mathrm{rpm} \sqrt{ } \mathrm{kW}$ is achievable with the novel high-strength materials described in the previous section.

The design of high-speed electrical machines is known to be very challenging due to the close coupling of the sciences involved [9]. To this end, the use of coupled multi-domain design environments is necessary to achieve a stable design, such as the one used in this investigation, shown in Figure 5. In this environment, the electromagnetic, thermal and mechanical domains run in parallel so that any geometrical change can be instantly assessed from all the domains. Moreover, comprehensive detailed information about the materials used and their behavior at different temperatures, under different mechanical stresses and different excitations is included within the design environment, as is the information about the various manufacturing techniques.

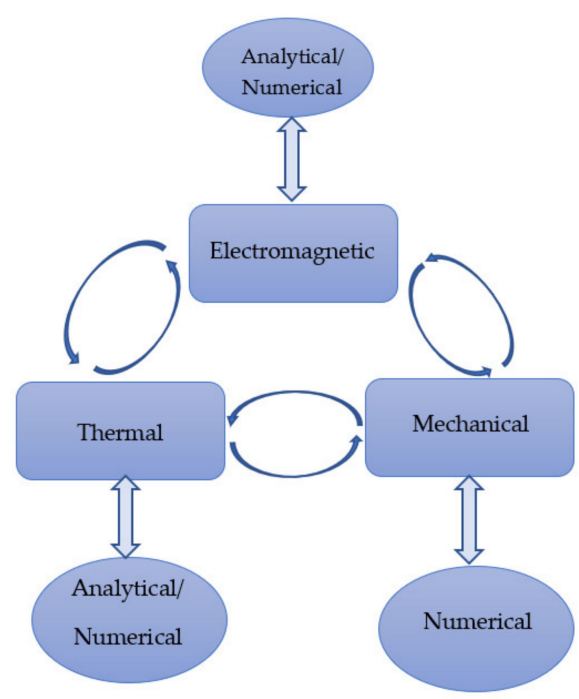

Figure 5. Multi-domain design procedure for high-speed induction motors.

\subsection{Multidomain High-Speed Induction Machine Design Procedure}

With the electromagnetic, thermal and mechanical domains coupled, the induction machine is designed following the high speed laminated rotor induction machine design procedure described in detail in [9] and reproduced in Figure 6. As suggested in the same paper, drop-shaped rotor bars are utilized to maximize the power density while avoiding high stress concentrations on the rotor laminations.
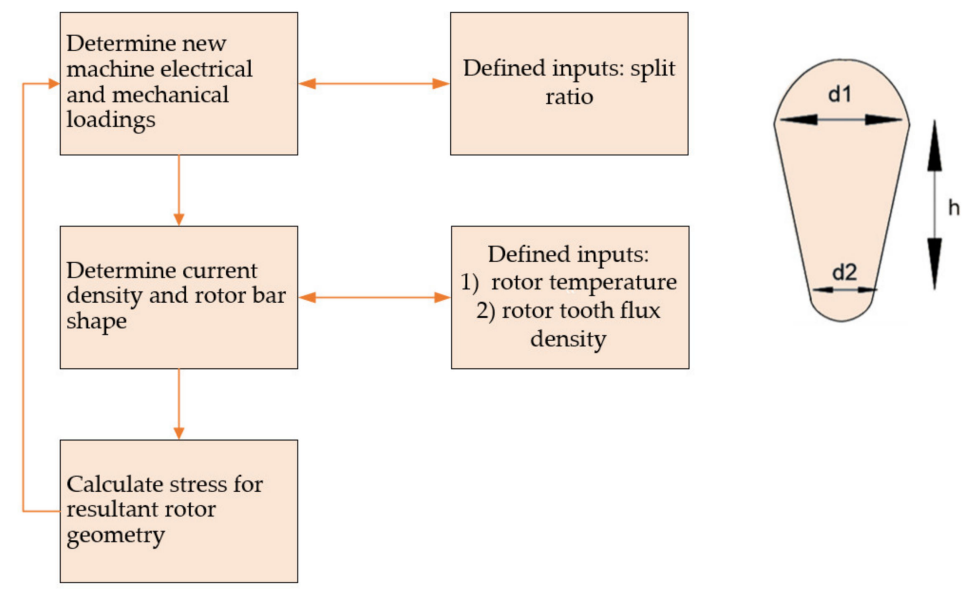

Figure 6. Suggested design methodology (left) and drop-shaped bar used (right).

The multi-domain design methodology for maximizing the power density of laminated rotor HSIM (High Speed Induction Machine) is described in Figure 6. Basically, for each 
rotor diameter considered, the various combinations of electrical/magnetic loadings, which correspond to the particular diameter, are evaluated. The different electrical/magnetic loadings correspond to different airgap flux-densities, turn number as well as rotor bar current, as described in [9].

Based on the (i) rotor bar current, (ii) maximum rotor temperature and (iii) a predefined flux-density in the rotor teeth, the drop-shaped bar can be automatically sized and the resultant mechanical stress calculated. The procedure is repeated for different rotor diameters, to identify the most feasible rotor design, with reduced stress and an acceptable temperature rise, as one case shown in Figure 7.
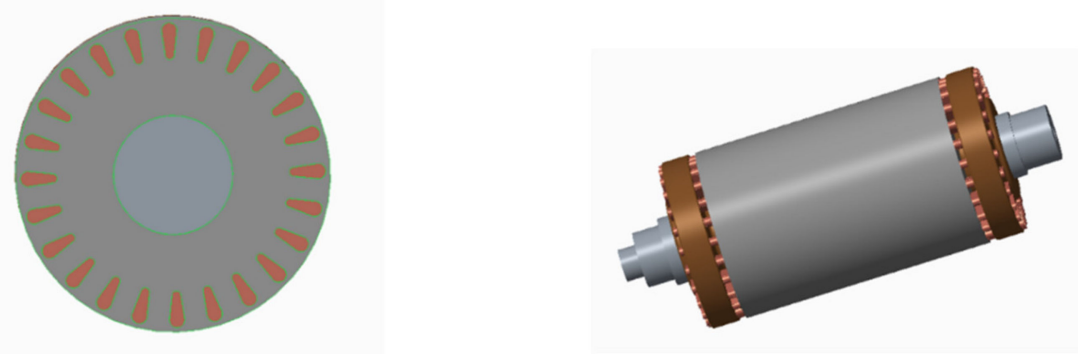

Figure 7. Resultant induction machine rotor geometry.

\subsection{Mechanical Design Aspects}

Since this paper treats the exploitation of novel high-strength materials to increase the power-speed capability of a laminated rotor induction machine, in this section, greater focus is expended on the rotor mechanical design aspects of the machine. Given that the machine targets a very high $\mathrm{rpm}_{\sqrt{ } \mathrm{kW}}$, the machine is designed using the novel highstrength materials described in Section 2, but importantly, avoiding any material that uses rare-earth materials such as Cobalt Iron laminations, since this paper aimed to develop a rare-earth-free machine solution.

Table 4 summarizes the materials considered, including the high strength HXT780T, which is the best-in-class commercially available high-strength $\mathrm{SiFe}$, with a yield strength of $850 \mathrm{MPa}$. For the rotor bars, $\mathrm{CuCrZr}$ is considered, given its high yield strength (500 MPa) and good conductivity ( $80 \%$ IACS). For the end-rings, where a higher mechanical stress is expected with respect to the bars, the Beryllium-free $\mathrm{CuNiSiCr}$ alloy, with a yield strength of $820 \mathrm{MPa}$ and a conductivity of $27 \%$ IACS, is considered.

Table 4. Materials for $150 \mathrm{~kW}, 50 \mathrm{krpm}$ induction motor.

\begin{tabular}{|c|c|c|c|}
\hline Items & Materials & Yield Strength (MPa) & $\begin{array}{c}\text { Mass-Density } \\
\left(\mathrm{kg} / \mathrm{m}^{3}\right)\end{array}$ \\
\hline Shaft & Stainless, '17-4PH' & 1000 & 7750 \\
\hline Rotor Laminations & SiFe, 'HXT780T' & 850 & 8950 \\
\hline Cu-bar & $\mathrm{CuCrZr}$ & 500 & 8933 \\
\hline End-ring & CuNiSiCr & 820 & 8700 \\
\hline
\end{tabular}

\subsubsection{Laminations}

The rotor laminations and the rotor end-rings can be simplified as a rotating hollow disk for first-level stress analysis. For a rotating hollow disk, the hoop stress and radial stress distributions can be calculated using the following equations [10]:

$$
\begin{aligned}
\sigma_{r} & =A-\frac{B}{r^{2}}-(3+v) \frac{\rho \omega^{2} r^{2}}{8} \\
\sigma_{H} & =A+\frac{B}{r^{2}}-(1+3 v) \frac{\rho \omega^{2} r^{2}}{8}
\end{aligned}
$$


where ' $\sigma_{r}$ ' and ' $\sigma_{H}$ ' are the stress in the radial and hoop direction of the disk, ' $A$ ' and ' $B$ ' are constant values at the inner and outer radii of the hollow disk, ' $\omega$ ' is the rotating speed of the disk, ' $r$ ' is the radius of the disk and ' $v$ ' and ' $p$ ', are the material Poisson ratio and mass-density, respectively, of the material.

Using the simple hollow disk equations, Figure 8 shows the stress distribution of the rotor laminations and end-rings at the rotational speed of $50 \mathrm{krpm}$. As can be seen from the figure, the maximum stress for the lamination is around $400 \mathrm{MPa}$ and occurs at the inner edge of the disk. Due to the different material mass-density and Poisson ratio, the stress at the end-ring inner diameter is slightly higher, around $450 \mathrm{MPa}$. Importantly, both the initially obtained stress values for the lamination and the end-ring indicate that, at the diameter and speed targeted, the use of special materials with a markedly higher yield strength with respect to the conventional materials is required.

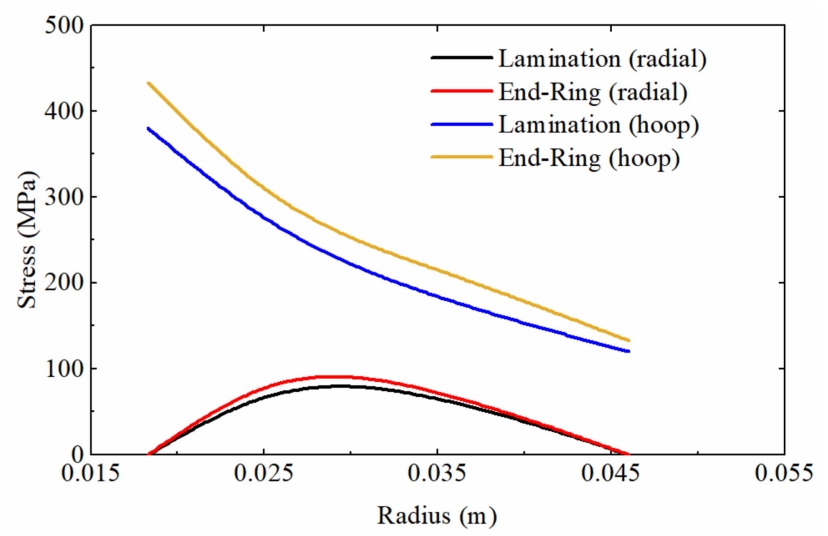

Figure 8. Lamination and end-ring stress calculated from rotating hollow disk equations.

For an Induction Machine's rotor, the lamination stack also holds the rotor bars. Due to the limited mechanical strength of the long thin bars, the lamination stack provides retaining support against the centrifugal forces of the copper bars in the radial direction. This generates extra stress in the laminate disk, beyond that calculated by simply using Equation (1).

The detailed distribution of stress including the centrifugal load and the load due to the bars is obtained using Finite Element Analysis (FEA). Figure 9a shows the stress distribution of the lamination at $50 \mathrm{krpm}$. The copper bars' masses are added to the slots on the laminate disk, which allows the total centrifugal force loads to be simulated. From Figure 9a it can be observed that the maximum stress in the lamination occurs at the bottom of the slots due to the extra centrifugal loading from the bars and stress concentration at inner corner of opening, which is different compared to the hollow disk where the maximum stress occurs at the inner surface of the disk. The maximum stress in the lamination is $687 \mathrm{MPa}$, which is an over $75 \%$ increase with respect to the hollow disk only, although still below the yield strength of the HXT780T material by over $20 \%$. Moreover, compared to Figure 8 ,. the extra load from the rotor bars also significantly increases the stress level at the inner surface of the hollow disk. 


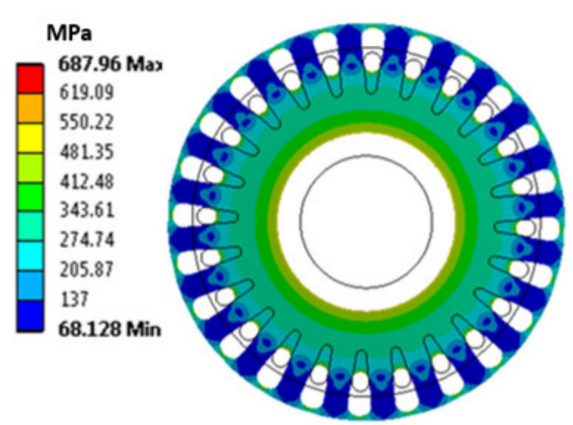

(a) stress distribution

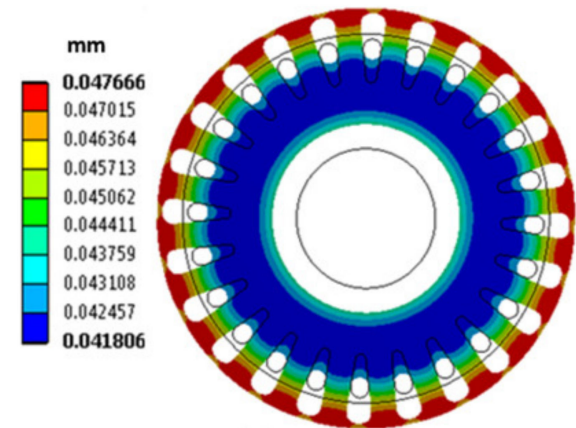

(b) deformation

Figure 9. Stress distribution and deformation in HXT780T lamination including centrifugal and copper bar load.

Figure $9 \mathrm{~b}$ shows the deformation of the lamination under rotation. As expected, maximum deformation occurs at the outer surface of the lamination. The radial growth of the lamination at $50 \mathrm{krpm}$ is less than $0.047 \mathrm{~mm}$, which is less than $10 \%$ of the airgap of the machine.

The natural frequencies of the laminations are also predicted using the FEA method. Figure 10 shows the first and second mode shapes of the laminate disk. The first mode shape occurs at a frequency of $12,248 \mathrm{~Hz}$ and is much higher than the machine max operating speed of machine $50 \mathrm{krpm}(\sim 833 \mathrm{~Hz})$.

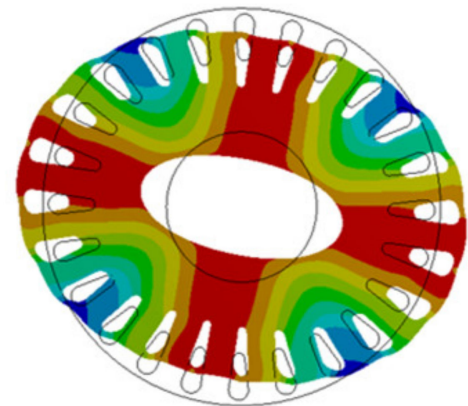

(a) 1st mode shape @12,248 Hz

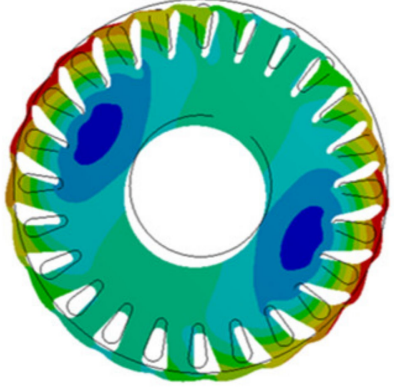

(b) 2nd mode shape @20,095 Hz

Figure 10. Natural frequency of lamination.

\subsubsection{End-Rings}

FEA is also used to predict the stress distribution on the $\mathrm{CuNiSiCr}$ end-rings when the $\mathrm{CuCrZr}$ bars are embedded within. Figure 11 shows the FEA results of stress and deformation of the disk at $50 \mathrm{krpm}$. The stress concentrations occur at the location where the bars are inserted with a maximum of $628 \mathrm{MPa}$, which is $40 \%$ higher with respect to the theoretical hollow disk, although still safely below the yield strength of the $\mathrm{CuNiSiCr}$ material. The maximum deformation of the disk at $50 \mathrm{krpm}$ is $0.07 \mathrm{~mm}$, as shown in Figure $9 b$.

\subsubsection{Rotor Bars and Rotor Cage}

An FEA simulation is carried out to understand the extent of the deformations when the rotor squirrel-cage rotates at $50 \mathrm{krpm}$, without the support of the laminations. As can be seen from Figure 12, the deformation of the $\mathrm{CuCrZr}$ bars is significantly large if the bars are only supported by the two end disks, under which circumstance the stress in the bar would well exceed the limitations of the material. However, for an induction machine rotor, all the conductors are inserted into the rotor lamination stack that supports the conductors, as shown earlier in Figure 7, and hence limits their deformation. The maximum deformation of lamination $(0.05 \mathrm{~mm}$, as determined from Figure 9), is applied as a boundary condition 
to the copper cage, as shown in Figure 13, in which case the maximum stress of the bar will be limited to around $400 \mathrm{MPa}$, yielding a mechanical design margin of 1.2.
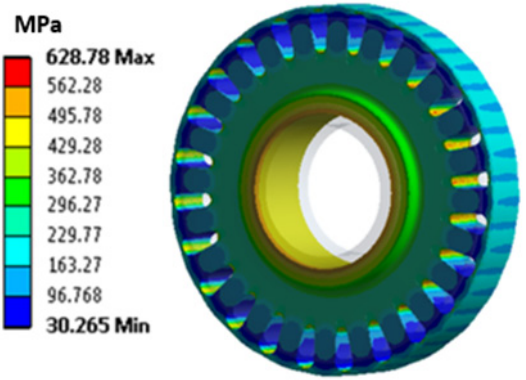

(a) stress distribution

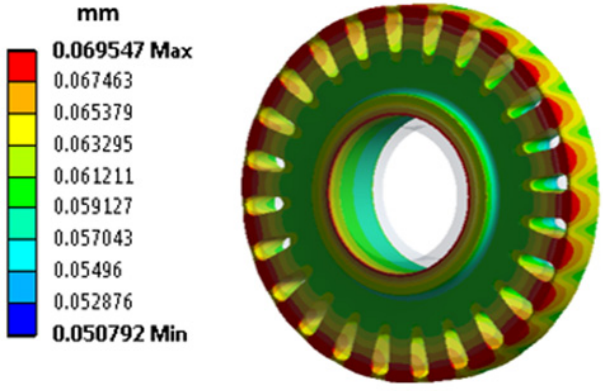

(b) deformation

Figure 11. Stress distribution and deformation in CuNiSiCr End-Ring including centrifugal and copper bar load.
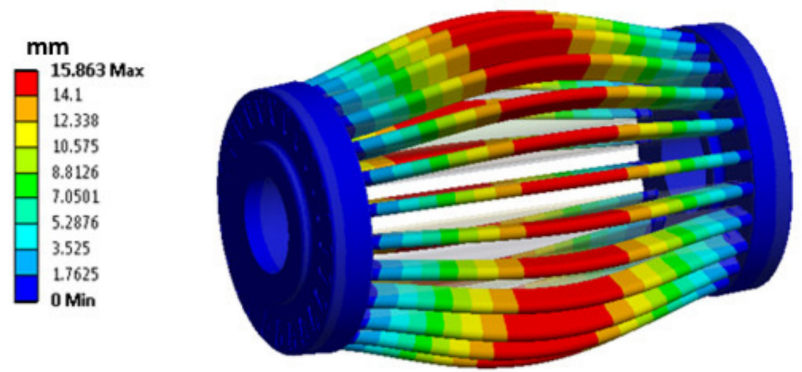

Figure 12. Rotor squirrel-cage deformation at $50 \mathrm{krpm}$, without support of laminations.
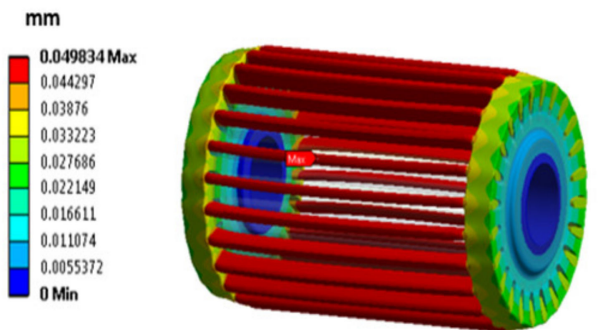

(a) deformation

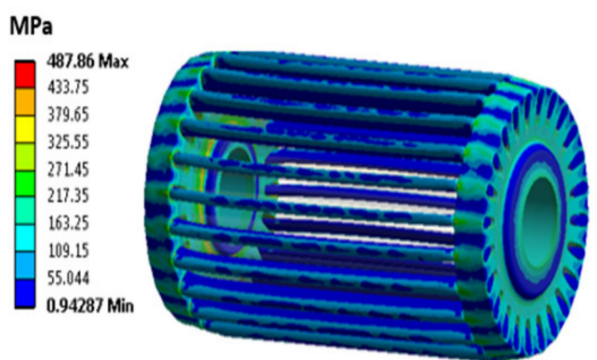

(b) stress distribution

Figure 13. Rotor squirrel-cage: (a) application of lamination deformations as a boundary condition, (b) resultant stress distribution in the cage.

\subsubsection{Rotor Dynamics}

In the first instance, the shaft itself is analyzed without the influence of the rotor core and the rotor cage. The first and second bending modes occur at the frequencies of 2839.9 and $7086.4 \mathrm{~Hz}$, respectively, well above the machine operating frequency of $833.3 \mathrm{~Hz}$. When the mass of the rotor laminations, copper-alloy bars and the two end-disks are added to the shaft, the natural frequency corresponding to the first bending mode drops to $1565.9 \mathrm{~Hz}$, while the second bending mode frequency drops to $3563.8 \mathrm{~Hz}$, as shown in Figure 14a,b. 


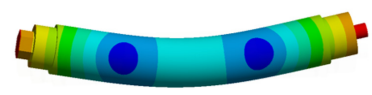

(a)

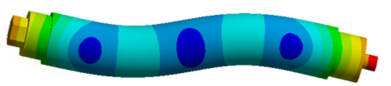

(b)

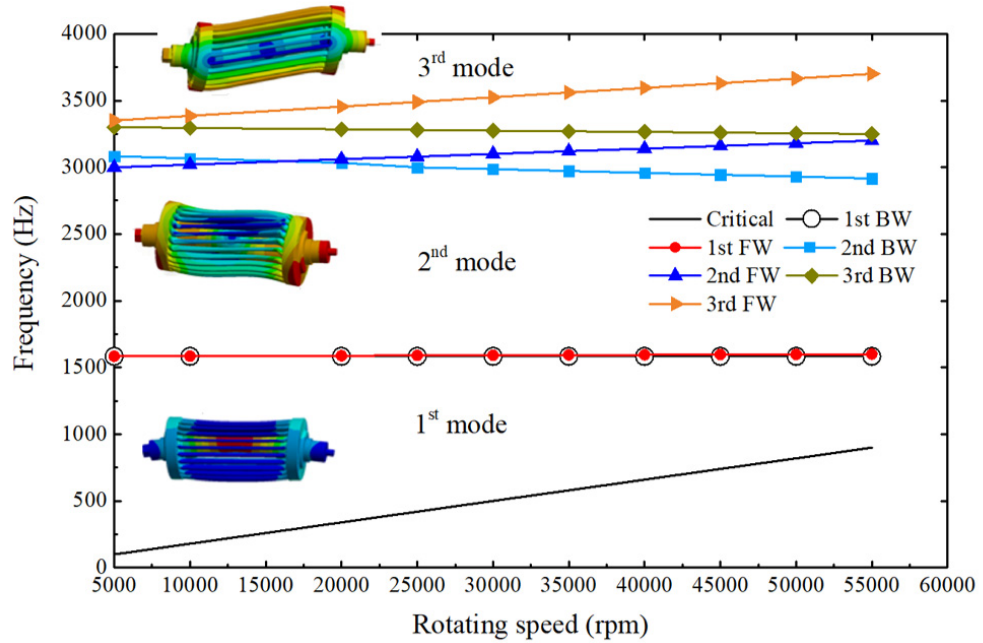

(c)

Figure 14. Campbell diagram considering the stiffening effect of the rotor cage. (a) 1st mode @ 1565.9 $\mathrm{Hz}$, (b) 2nd mode @3563.8 Hz, (c) the Campbell diagram of the rotor dynamic performance at the high stiffness bearing housing condition.

For more accurate analysis, the rotor assembly includes the shaft and the rotor cage. In this case, the cage, made of high-strength materials, apart from the well-known primary electromagnetic design function of generating torque, also serves an important mechanical design function of stiffening the rotor assembly.

\section{150 kW 50 krpm Induction Machine Development Aspects}

With the geometry and mechanical aspects considered, the machine is prototyped using the high strength materials. Due to low prototype volumes, the HXT780T laminations are laser cut from loose laminations. Similarly, the rotor bars and end-rings are wire-cut from solid $\mathrm{CuCrZr}$ and $\mathrm{CuNiSiCr}$ billets. For volume production, a punching tool can be used for the high strength laminations, while the alloys used for the rotor cage can be conveniently cast, or for the case of the rotor-bars an extrusion process can also be used. The cut laminations, rotor bars and end-rings are shown in Figure 15a. The bars are then inserted into the lamination stack and brazed using a high conductivity alloy. The rotor is then precision balanced for high-speed operation, as shown in Figure 15b.

Characterization is performed on a high-speed dynamometer in order to check the performance on-load. In Figure 16, the current and torque are compared to the FEA predictions at a frequent high-speed operation point of $40 \mathrm{krpm}$. For a given speed, the maximum difference to the predicted current is $11 \%$. 

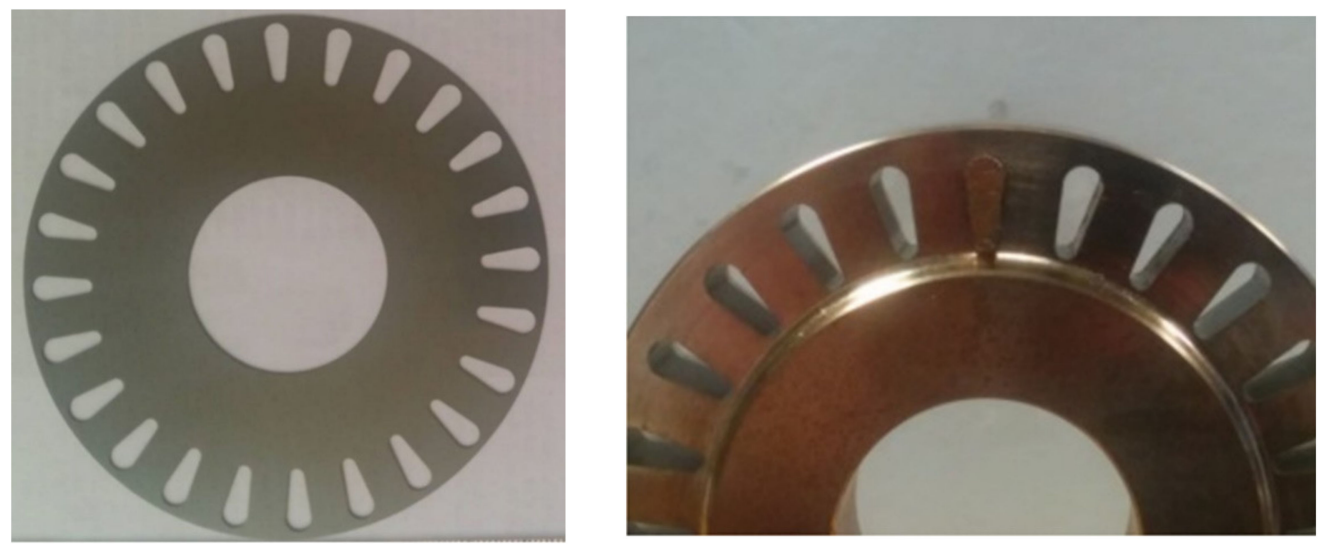

(a)

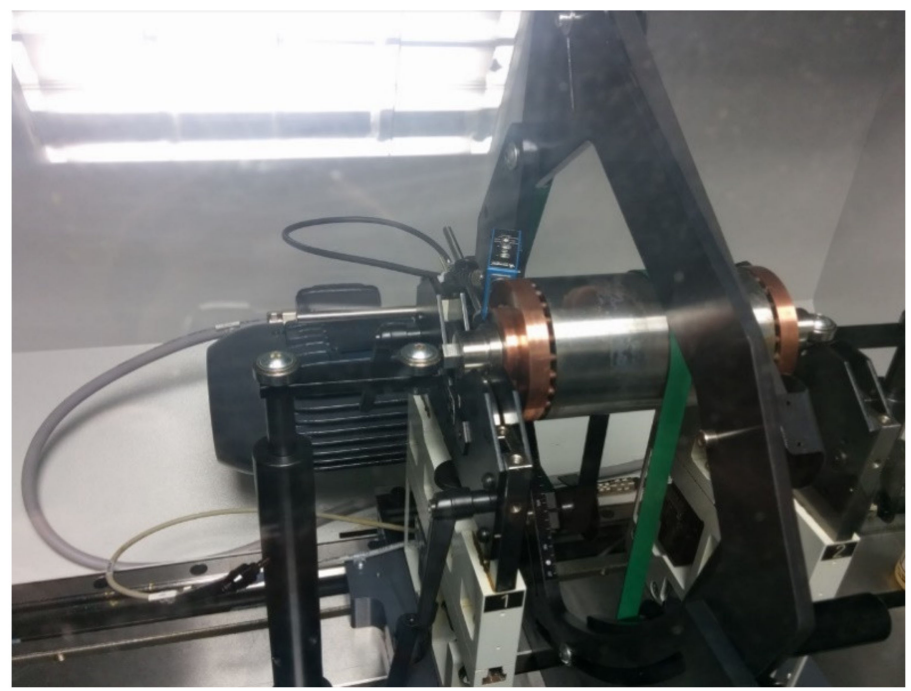

(b)

Figure 15. Prototyped high-speed induction machine for marine EAT application (a) lamination profile, (b) precision balancing of high-speed rotor assembly.

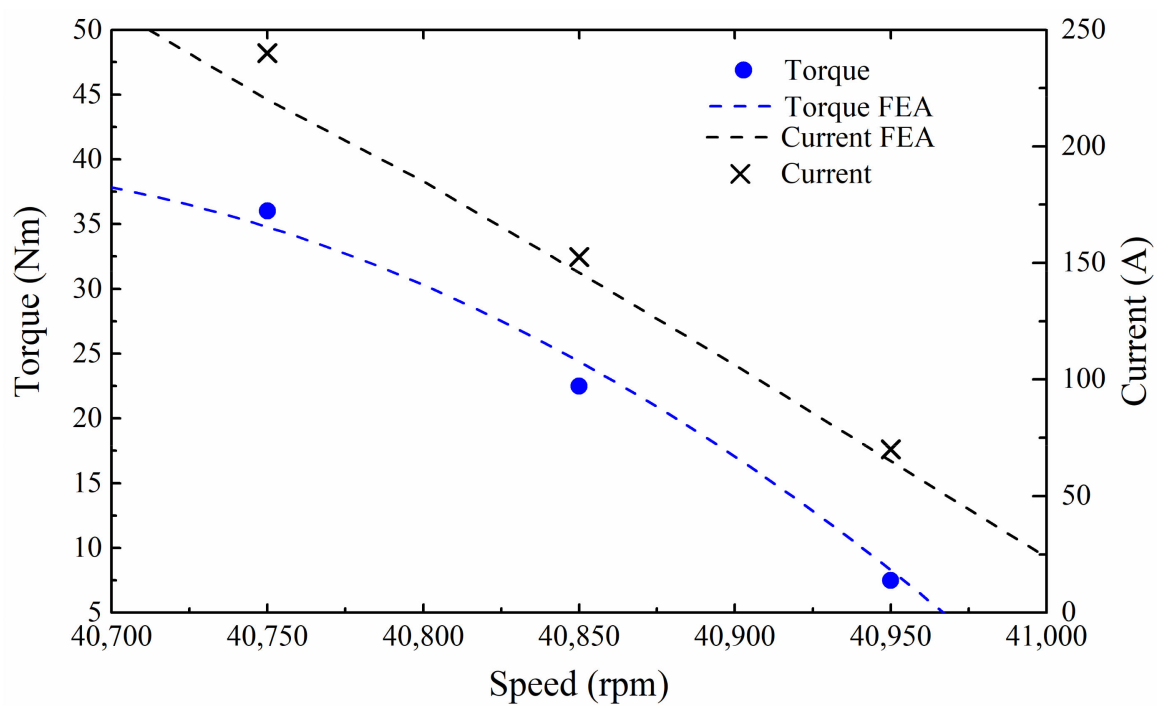

Figure 16. Comparison of performances at high speed. 


\section{Conclusions}

With the increasing drive to achieve higher power densities, the research, development and commercialization of such machines is at an all-time high. While the laminated rotor induction machine is a long-established, mature technology, it can sometimes be overlooked for higher power-speed product applications, as the achievable power-speed capability and peripheral speeds are traditionally lower with respect to those attained by the more common surface-PM topologies.

Recent developments in the IM's constituent materials include the development of environmentally friendly Be-free high-strength copper alloys and high-strength electrical steels, with yield strengths in excess of $800 \mathrm{MPa}$, around twice that of conventional steel.

This paper has described the mechanical design procedure for high-speed IMs, which can be summarized as:

- Start with an analytical calculation of stress in the lamination and end-ring at the maximum speed, assuming a circular hollow disk (no slots present) as the initial design.

- Complete detailed FEA analysis of the stress distribution and deformation for rotating laminations and end-rings including all the geometric features to guarantee a safety margin of mechanical strength even at the location where stress concentration occurs.

- $\quad$ Check the dynamic performances of the rotor at various conditions to ensure the critical speed is well above the machine operating speed.

By using the above mechanical design procedure within a multi-domain environment, and then exploiting the properties of the aforesaid novel electrical steels and copper alloys, this paper has shown that the laminated rotor IM can achieve higher $\operatorname{rpm} \sqrt{ } \mathrm{kW}$, similar to that typically associated with the SPM technology. This is an important result that can translate to an increase market uptake of high-power, high-speed drives, especially in applications where the mass (and cost) of rare-earths in SPM machines remains prohibitive, such as in waste heat recovery systems of high-horsepower engines.

Author Contributions: Investigation, F.Z., D.G. and Z.X.; conceptualization, D.G., Y.H., H.Z., W.H. and C.G.; supervision, C.G. All authors have read and agreed to the published version of the manuscript.

Funding: This research was funded by Ministry of Science \& Technology under National Key R\&D Program of China, under Grant 2021YFE0108600 and Ningbo Science and Technology Bureau under S\&T Innovation 2025 Major Special Programme with grant No. of 2019B10071.

Acknowledgments: The authors would like to thank Nippon Steel Sumitomo Metal for kindly providing the high strength laminations and to Cummins Generator Technologies for their support with high-speed motor research.

Conflicts of Interest: The authors declare no conflict of interest.

\section{References}

1. Van Millingen, R.; van Millingen, J. Phase shift torquemeters for gas turbine development and monitoring. In Turbo Expo: Power for Land, Sea, and Air; ASME: New York, NY, USA, 1991; p. V005T015A003.

2. Gerada, D.; Huang, X.; Zhang, C.; Zhang, H.; Zhang, X.; Gerada, C. Electrical machines for automotive electrically assisted turbocharging. IEEE/ASME Trans. Mechatron. 2018, 23, 2054-2065. [CrossRef]

3. Gerada, D.; Mebarki, A.; Brown, N.L.; Gerada, C.; Cavagnino, A.; Boglietti, A. High-speed electrical machines: Technologies, trends, and developments. IEEE Trans. Ind. Electron. 2014, 61, 2946-2959. [CrossRef]

4. Ikeda, M.; Sakabe, S.; Higashi, K. Experimental study of high speed induction motor varying rotor core construction. IEEE Trans. Energy Convers. 1990, 5, 98-103. [CrossRef]

5. Lateb, R.; Enon, J.; Durantay, L. High speed, high power electrical induction motor technologies for integrated compressors In Proceedings of the 2009 International Conference on Electrical Machines and Systems, Tokyo, Japan, 15-18 November 2009; pp. 1-5.

6. Senda, K.; Namikawa, M.; Hayakawa, Y. Electrical Steels for Advanced Automobiles-Core Materials for Motors, Generators and High-Frequency Reactors. Available online: https://www.jfe-steel.co.jp/en/research/report/004/pdf/004-12.pdf (accessed on 26 August 2021). 
7. Tanaka, I.; Yashiki, H. Magnetic and mechanical properties of newly developed high-strength nonoriented electrical steel. IEEE Trans. Magn. 2010, 46, 290-293. [CrossRef]

8. Yabumoto, M.; Kaido, C.; Wakisaka, T.; Kubota, T.; Suzuki, N. Electrical Steel Sheet for Traction Motor of Hybrid/Electric Vehicles. Available online: https:/ / www.nipponsteel.com/en/tech/report/nsc/pdf/103-18.pdf (accessed on 26 August 2021).

9. Gerada, D.; Mebarki, A.; Brown, N.L.; Bradley, K.J.; Gerada, C. Design aspects of high-speed high-power-density laminated-rotor induction machines. IEEE Trans. Ind. Electron. 2011, 58, 4039-4047. [CrossRef]

10. Hearn, E. Rings, discs and cylinders subjected to rotation and thermal gradients. In Mechanics of Materials 2; Elsevier Ltd.: Amsterdam, The Netherlands, 1997; pp. 117-140. 